\title{
Electrophonon resonances in a quasi-two-dimensional electron system
}

\author{
W. Xu, F. M. Peeters, and J. T. Devreese* \\ Departement Natuurkunde, Universiteit Antwerpen (UIA), Universiteitsplein 1, B-2610 Antwerpen, Belgium
}

(Received 16 March 1992; revised manuscript received 8 June 1992)

\begin{abstract}
When the energy difference between two electric subbands in a quasi-two-dimensional electron system equals a LO-phonon energy, resonant scattering will occur. This leads to an enhancement of the scattering rate and, consequently, to a suppression of the conductivity. Changing the energy difference between the electric subbands (e.g., through a gate) leads to a series of electrophonon resonances in the conductivity. A detailed study is made of this effect for different confinement potentials. We found that the scattering processes where the emission of a phonon is involved are very important for the electrophonon resonance and that the size of the effect decreases with increasing temperature.
\end{abstract}

\section{INTRODUCTION}

For temperatures $T>50 \quad \mathrm{~K}, \quad$ scattering with longitudinal-optical (LO) phonons is the dominant scattering process limiting the mobility of electrons in a polar semiconductor ${ }^{1}$ like GaAs. The effect of the electron LO-phonon coupling on the electrical properties is influenced by the fact that the electrons are confined to move in two dimensions (2D). For example, during a scattering event the momentum in the $z$ direction is no longer conserved. This leads to an enhancement of the one-electron effective electron-phonon interaction ${ }^{2}$ and consequently to a lowering of the mobility. Recently ${ }^{3}$ it was shown that the one-electron LO-phonon scattering limited mobility in $2 \mathrm{D}$ is related to the three-dimensional (3D) result by the relation $\mu_{2 D}(\alpha)=\mu_{3 D}(3 \pi \alpha / 4)$, where $\mu_{n D}$ is the mobility of an $n$-dimensional system and $\alpha$ the electron-phonon coupling constant. Because $3 \pi / 4$ $\simeq 2.36>1$, the high-temperature mobility $(\mu \sim 1 / \alpha)$ of an ideal one-electron 2D system is reduced by a factor 0.42 as compared to the corresponding $3 \mathrm{D}$ system. In real systems the situation is more complicated: (1) We have a two-dimensional gas of electrons (2DEG) such that many-particle effects ${ }^{4}$ such as occupation effects, screening, electron-electron scattering, etc., are important and (2) the 2DEG has a finite width which will reduce the effective electron-phonon interaction.

In most papers on electron transport in a quasi-twodimensional electron gas (Q2DEG), one assumes that only one electric subband is occupied. In the present paper we are interested in effects which are a consequence of occupation of several electric subbands. In such a case we predict that each time the energy difference of two electric subbands equals the energy of a LO phonon $\left(\hbar \omega_{\text {LO }}\right)$, there will be resonant scattering which leads to a reduction of the mobility. Consequently, the conductivity is expected to oscillate as a function of energy difference between the electric subbands.

In the present paper we investigate Q2DEG with different confinement potentials: (1) the parabolic well and (2) the square well, which both have the advantage that all subbands can be included in the calculation; and (3) the triangular well, which is often used to model het-

erostructures. By changing the strength of these potentials, the energy difference between electric subbands can be altered. In a real device the relative position of the electric subbands can be altered by applying a bias field on, e.g., a heterostructure. This was recently realized in Ref. 5 in a graded quantum well, which is schematically depicted in Fig. 1(a). One applies a small electric field $F_{x}$ along the interface in the $x$ direction and a bias field $F_{z}$ perpendicular to the layer in the $z$ direction. By changing the bias field and observing the variation of the conductivity in the $x$ direction, this electrophonon resonance effect was measured. The energy levels between two different electric subbands are altered by changing $F_{z}$. To measure the electrophonon resonance effect, $F_{z}$ should be changed around the value $F_{z}=n F_{0} \quad\left(F_{0}=92.5\right.$ $\mathrm{kV} / \mathrm{cm}$ for $\mathrm{GaAs})$. The corresponding energy diagram for the two lowest electric subbands is shown in Fig. 1(b) for two different values of the electric field $F_{z}$.

This new electrophonon resonance effect is the electrical equivalent of the magnetic magnetophonon resonance effect. A recent review of the magnetophonon and electrophonon resonance effects was given ${ }^{6}$ by Peeters and Devreese. The magnetophonon resonance effect was pre-

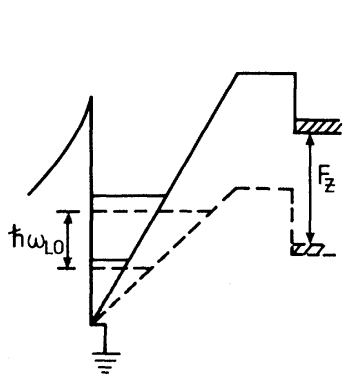

(a)

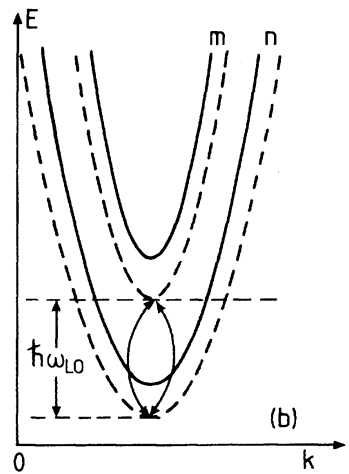

FIG. 1. (a) Applying a bias field $\left(F_{Z}\right)$, the energy separation between two different electric subbands can be altered. (b) Electrophonon resonance occurs when the energy difference between two electric subbands equals a LO-phonon energy $\hbar \omega_{\mathrm{LO}}$. 
dicted by Gurevich and Firsov ${ }^{7}$ and has been observed experimentally, ${ }^{8-11}$ e.g., in the magneto resistance of a polar semiconductor ${ }^{8}$ and in the subband splitting of a metal-insulator-semiconductor (MIS) structure. ${ }^{9}$ Under the action of a magnetic field, the magnetophonon resonance occurs when the condition $N \hbar \omega_{c}=\hbar \omega_{L O}$ is satisfied ( $\omega_{c}$ is the cyclotron frequency). The magnetophonon resonance has been studied theoretically, ${ }^{12}$ which is based on a force-balance equation ${ }^{13}$ for the magnetotransport in a 2D-electron system. Less much work has been done on the electrophonon resonance effect (EPR). ${ }^{9,14-16}$ In Ref. 9 the subband splitting in a MIS structure, i.e., an $n$ inversion layer on $p$-Insb, was electrically tuned by varying the electron density. The electrophonon resonance effect was then observed when a strong quantizing magnetic field perpendicular to the interface is applied in this manner, providing a "zero-dimensional" energy-level structure. In Ref. 15 a Monte Carlo simulation was performed for a quantum wire in high field transport, and resonant intersubband optic-phonon scattering was found when the subband spacing is equal to the LO-phonon frequency. The electrophonon resonance was studied ${ }^{16}$ for a graded quantum well by applying a momentum-balance equation approach which was based on the Boltzmann equation. Recently, Kastalsky et al. presented ${ }^{5}$ the experimental evidence of the electrophonon resonance in a $\mathrm{AlGaAs} / \mathrm{Al}_{x} \mathrm{Ga}_{1-x}$ As triangular quantum well (TQW).

In the present paper we study the electrophonon resonance effect in different two-dimensional semiconductor systems. The aim of this study is (1) to study the electrophonon resonance effect in a Q2DEG with different confinement potentials and (2) to calculate the conductivity as a function of the confinement-potential parameters. We limit ourselves to a one-electron system because at the high temperatures we are interested in, FermiDirac statistics is of minor importance. In Sec. II the scattering of electrons by LO phonons is discussed. We will neglect all other scattering mechanisms which will be of secondary importance for the considered temperatures. The numerical results for the conductivity near the electrophonon resonant scattering is discussed in detail in Sec. II. In Sec. III we present the detailed calculation of the conductivity versus the confinements as function of (1) the width of the quantum well for the square well case, (2) the electric field for the triangular-well case, and (3) the resonant frequency of the harmonic oscillator for the parabolic-well case at different lattice temperatures. An approximation to calculate the conductivity near the electrophonon resonances is presented. Also, the influence of electron LO-phonon emission and absorption scattering is studied, as well as the contribution from the difference electric subbands on this electrophononresonance effect. Our conclusions are summarized in Sec. IV.

\section{THE SCATTERING OF ELECTRONS BY LO PHONONS IN A QUASI-2D SYSTEM}

In a quasi-two-dimensional electron system, the electron motion along one direction (taken along the $z$ axis) is quantized into discrete levels and the energy spectrum becomes

$$
E_{n}(\mathbf{k})=E_{n}+E_{\mathbf{k}}
$$

where

$$
E_{\mathbf{k}}=\left(\hbar^{2} / 2 m^{*}\right)\left(k_{x}^{2}+k_{y}^{2}\right)
$$

is the kinetic energy for free-electron motion in the $x y$ plane and $E_{n}$ are the electric quantum levels arising from confinement with corresponding wave functions $\psi_{n}(z)$. Ridley studied ${ }^{17}$ the scattering rate of electron LOphonon coupling through applying the momentumconservation approximation (MCA). In this present paper we will not use this approach to calculate the scattering rate. Applying Fermi's golden rule to the Fröhlich Hamiltonian, we find that the scattering rate for an electron which is in subband $n$ and has a kinetic energy $x=E_{\mathrm{k}} / \hbar \omega_{\mathrm{LO}}$ is given by

$$
\begin{aligned}
\lambda_{n m}(x)= & \alpha \frac{\omega_{\mathrm{LO}}}{L_{0}}\left[\begin{array}{c}
N_{0} \\
N_{0}+1
\end{array}\right] \Theta\left[x\left(x \pm 1-x_{m}+x_{n}\right)\right] \\
& \times \int_{-\infty}^{+\infty} d q_{z} \frac{G_{m n}^{2}\left(q_{z}\right)}{\left(q_{z}^{4}+b_{ \pm} q_{z}^{2}+a_{ \pm}^{2}\right)^{1 / 2}},
\end{aligned}
$$

where $x_{n}=E_{n} / \hbar \omega_{\mathrm{LO}}$ is the subband energy in units of the LO-phonon energy $\left(\hbar \omega_{\mathrm{LO}}\right), L_{0}=\left(\hbar / 2 m^{*} \omega_{\mathrm{LO}}\right)^{1 / 2}$ is taken as the unit of length which equals $39.5 \AA$ for GaAs,

$$
N_{0}=1 /\left(e^{\hbar \omega_{\mathrm{LO}} / k_{B} T}-1\right)
$$

is the LO-phonon occupation number, $a_{ \pm}=\mid x_{m}$ $-x_{n} \mp 1 \mid / L_{0}^{2}$

$$
b_{ \pm}=4\left[x \pm \frac{1}{2}-\left(x_{m}-x_{n}\right) / 2\right] / L_{0}^{2},
$$

$\Theta(x)$ is the $\Theta$ function, $\alpha$ is the Fröhlich coupling constant, which is 0.068 for GaAs, and the upper (lower) sign refers to absorption (emission) of a LO phonon. In Eq. (2a) we defined the form factor

$$
G_{m n}\left(q_{z}\right)=\int d z \psi_{m}^{*}(z) \psi_{n}(z) e^{i q_{z} z} .
$$

For the special cases of (i) zero energy, i.e., $\tilde{x}=x=0$, and (ii) at resonance, i.e., $\widetilde{x}=x=x_{m}-x_{n} \mp 1$, Eq. (2a) is defined as

$$
\lambda_{n m}(\tilde{x})=\alpha \frac{\omega_{\mathrm{LO}}}{L_{0}}\left[\begin{array}{c}
N_{0} \\
N_{0}+1
\end{array}\right] \int_{-\infty}^{+\infty} d q_{z} \frac{G_{m n}^{2}\left(q_{z}\right)}{q_{z}^{2}+a_{ \pm}} .
$$

In order to calculate the scattering, we have to specify the confinement potential. We consider three different kinds of confinement potentials.

\section{A. The parabolic well}

One of the main merits of the parabolic-well case $V(z)=m^{*} \omega_{0} z^{2} / 2$ is that all electric subbands can be included in the calculation. The wave functions are ${ }^{18}$

$$
\psi_{n}(z)=(2 \pi)^{-1 / 4} \frac{\widetilde{\omega}^{1 / 4}}{L_{0}^{1 / 2}} \frac{1}{\sqrt{2^{n} n !}} e^{-\xi^{2} / 2} H_{n}(\xi),
$$


with the corresponding energies

$$
E_{n}=\hbar \omega_{0}\left(n+\frac{1}{2}\right), \quad n=0,1,2, \ldots,
$$

and

$$
x_{n}=E_{n} / \hbar \omega_{\mathrm{LO}}=\widetilde{\omega}\left(n+\frac{1}{2}\right),
$$

where $\omega_{0}$ is the resonant frequency of the harmonic oscillator, $\widetilde{\omega}=\omega_{0} / \omega_{\mathrm{LO}}, \xi=\widetilde{\omega}^{1 / 2} z /\left(\sqrt{2} L_{0}\right)$, and $H_{n}(x)$ are the Hermite polynomials.

The scattering rate is given by

$$
\begin{aligned}
& \lambda_{n m}(x)= \frac{2 \alpha \omega_{\mathrm{LO}}}{\widetilde{\omega}^{1 / 2}}\left[\begin{array}{c}
N_{0} \\
N_{0}+1
\end{array}\right] \Theta\{x[x \pm 1-\widetilde{\omega}(m-n)]\} \\
& \times \frac{q !}{p !} \int_{0}^{\infty} d y \frac{e^{-y^{2}} y^{2(p-q)}}{\left(y^{4}+b L_{0}^{2} y^{2} / \widetilde{\omega}+a^{2} L_{0}^{4} / \widetilde{\omega}^{2}\right)^{1 / 2}} \\
& \times\left[L_{p}^{p-q}\left(y^{2}\right)\right]^{2},
\end{aligned}
$$

where $p=\max \{m, n\}, \quad q=\min \{m, n\}, \quad a=[\widetilde{\omega}(m-n)$ $\mp 1] / L_{0}^{2}$, and

$$
b=4\left[x \pm \frac{1}{2}-\widetilde{\omega}(m-n) / 2\right] / L_{0}^{2},
$$

and

$$
L_{n}^{m}(x)=\sum_{i=0}^{n}(-1)^{i}\left(\begin{array}{c}
n+m \\
n-i
\end{array}\right) x^{i} / i !
$$

are the Laguerre polynomials.

\section{B. The square well}

For a square well of infinite height, the eigenvalues

$$
x_{n}=n^{2} E_{0}, \quad n=1,2,3, \ldots
$$

and envelope wave functions are known analytically, ${ }^{19}$

$$
\psi_{n}(z)=\left[\frac{2}{L}\right]^{1 / 2} \sin \left(n \pi \frac{z}{L}\right)
$$

where $L$ is the width of the square well and the energy difference between two electric subbands $n$ and $m$ is $E_{0}\left|m^{2}-n^{2}\right| \hbar \omega_{\text {LO }}$, with $E_{0}=\left(\pi L_{0} / L\right)^{2}$.

The scattering rate for an electron in subband $n$ with an in-plane kinetic energy $x=E_{\mathrm{k}} / \hbar \omega_{\mathrm{LO}}$ becomes

$$
\begin{aligned}
\lambda_{n m}\left(\frac{E_{\mathrm{k}}}{\hbar \omega_{\mathrm{LO}}}\right)= & \frac{\pi^{2} \alpha}{2} \frac{\omega_{\mathrm{LO}}}{\sqrt{E_{0}}}\left[\begin{array}{c}
N_{0} \\
N_{0}+1
\end{array}\right] \\
& \times \Theta\left\{x\left[x \pm 1-E_{0}\left(m^{2}-n^{2}\right)\right]\right\} \\
& \times \sum_{i=1}^{3} I_{n m}^{(i)}\left[\frac{E_{\mathrm{k}}}{\hbar \omega_{\mathrm{LO}}}\right]
\end{aligned}
$$

where

$$
\begin{aligned}
& I_{n m}^{(1)}(x)=\left[4 B_{n m}^{2}(x)+2 B_{n m}(x) \pi^{2}(m+n)^{2}+\frac{\pi^{4}}{4}(m+n)^{4}-4 A_{n m}^{2}(x)\right]^{-1 / 2}, \\
& I_{n m}^{(2)}(x)=\left[4 B_{n m}^{2}(x)+2 B_{n m}(x) \pi^{2}(m-n)^{2}+\frac{\pi^{4}}{4}(m-n)^{4}-4 A_{n m}^{2}(x)\right]^{-1 / 2}\left(1+\delta_{m n}\right), \\
& I_{n m}^{(3)}(x)=-\frac{\pi^{3}}{2} m^{2} n^{2} \int_{0}^{\pi} d \theta \frac{g_{n m}(x, \theta)\left[A_{n m}(x) \cos \theta+B_{n m}(x)\right]^{1 / 2}}{\left[A_{n m}(x) \cos \theta+B_{n m}(x)+\pi^{2}(m+n)^{2} / 4\right]^{2}\left[A_{n m}(x) \cos \theta+B_{n m}(x)+\pi^{2}(m-n)^{2} / 4\right]^{2}},
\end{aligned}
$$

with

$$
g_{n m}(x, \theta)=1+\eta \exp \left[-2 \sqrt{A_{n m}(x) \cos \theta+B_{n m}(x)}\right]
$$

where $\eta=1$ for $m+n=3,5,7, \ldots$,

$$
\text { and } \eta=-1 \text { for } m+n=2,4,6, \ldots
$$

and

$$
\begin{aligned}
& A_{n m}(x)=\pi^{2}\left\{x\left[x \pm 1-E_{0}\left(m^{2}-n^{2}\right)\right]\right\}^{1 / 2} /\left(2 E_{0}\right), \\
& B_{n m}(x)=\pi^{2}\left[x \pm \frac{1}{2}-E_{0}\left(m^{2}-n^{2}\right) / 2\right] /\left(2 E_{0}\right) .
\end{aligned}
$$

\section{The triangular well}

For the half-triangular-well case $\mathrm{e}^{20}$ the eigenfunctions are Airy functions ${ }^{21}$

$$
\psi_{n}(z)=A i\left[\left(\frac{2 m^{*} e F_{s}}{\hbar^{2}}\right]^{1 / 3}\left[z-\frac{E_{n}}{e F_{s}}\right]\right],
$$

where the eigenenergies are approximately given by

$E_{n}=\left(\hbar^{2} e^{2} F_{s}^{2} / 2 m^{*}\right)^{1 / 3}\left[3 \pi\left(n+\frac{3}{4}\right) / 2\right]^{2 / 3}, \quad n=0,1,2, \ldots$.

The exact eigenvalues can be obtained by replacing $n+\frac{3}{4}$ by 0.7587 and 1.7540 for the two lowest subbands, respectively. In practical calculation one is often forced to approximate Eq. (7a) through variational wave functions ${ }^{21}$ in order to keep the numerical calculations tractable. For the lowest subband $(n=0)$ and the first excited subband $(n=1)$, the wave functions are ${ }^{22-24}$ respectively,

$$
\psi_{0}(z)=\left(b_{0}^{3} / 2\right)^{1 / 2} z e^{-b_{0} z / 2}
$$


and

$$
\psi_{1}(z)=A\left(2 / b_{0}^{3}\right)^{1 / 2} z(1-B z) e^{-b_{1} z / 2},
$$

with

$$
b_{0}=2\left(3 e F_{s} m^{*} / 2 \hbar^{2}\right)^{1 / 3},
$$

$b_{1} \simeq 0.754 b_{0}, A \simeq 0.47 b_{0}^{3}$, and $B \simeq 0.292 b_{0}$, where $F_{s}$ is the "electric field" defining the well $V(z)=e F_{s} z(z>0)$, $\infty(z<0)$. For convenience we introduce the unit of electric field

$$
F_{0}=\omega_{\mathrm{LO}}\left(2 m^{*} \hbar \omega_{\mathrm{LO}}\right)^{1 / 2} / e
$$

which equals $92.5 \mathrm{kV} / \mathrm{cm}$ for GaAs.

The different scattering rates $\lambda_{n m}(x)$ corresponding to the two lowest subbands are now given by

$$
\begin{aligned}
\lambda_{00}^{ \pm}(x)= & 2 \alpha b_{0} L_{0} \omega_{\mathrm{LO}}\left[\begin{array}{c}
N_{0} \\
N_{0}+1
\end{array}\right] \Theta[x(x \pm 1)] \\
& \times \int_{0}^{\pi / 2} d \theta \frac{\cos ^{6} \theta}{D_{ \pm}(1,1,1,0,0, x, \theta)},
\end{aligned}
$$

with

$$
x_{0}=E_{0} / \hbar \omega_{\mathrm{LO}}=b_{0}^{2} L_{0}^{2} A_{0},
$$

$A_{0}=0.70812, F_{s} / F_{0}=b_{0}^{3} L_{0}^{3} / 6$, and the function

$$
\begin{aligned}
& D_{ \pm}^{2}\left(\gamma, \delta, \eta, n^{\prime}, n, x, \theta\right)=\gamma b_{0}^{4} L_{0}^{4} \sin ^{4} \theta+\delta b_{0}^{2} L_{0}^{2}\left[x \pm \frac{1}{2}+\left(x_{n^{\prime}}-x_{n}\right) / 2\right] \sin ^{2} 2 \theta+\eta \cos ^{4} \theta \\
& \lambda_{11}^{ \pm}(x)=2 \alpha b_{0} L_{0} \omega_{\mathrm{LO}} B_{0}\left[\begin{array}{c}
N_{0} \\
N_{0}+1
\end{array}\right] \Theta[x(x \pm 1)] \int_{0}^{\pi / 2} d \theta \frac{\cos ^{6} \theta\left(1+a_{3} \cos ^{2} \theta+a_{4} \cos ^{4} \theta+a_{5} \sin ^{2} 2 \theta\right)}{D_{ \pm}\left(a_{1}, a_{2}, 1,1,1, x, \theta\right)}
\end{aligned}
$$

with $\quad B_{0}=7.251515, \quad a_{1}=0.323210, \quad a_{2}=0.568516$, $a_{3}=0.751937, a_{4}=-1.525256, a_{5}=-0.899859$, and

$$
\begin{aligned}
& x_{1}=E_{1} / \hbar \omega_{\mathrm{LO}}=b_{0}^{2} L_{0}^{2} A_{1}, \\
& A_{1}=1.238073 .
\end{aligned}
$$

The above expressions $(8 a)-(8 b)$ are the intralevel scattering rates. The interlevel scattering rates are

$$
\begin{aligned}
\lambda_{10}^{ \pm}(x)= & 2 \alpha b_{0} L_{0} \omega_{\mathrm{LO}} C_{0}\left[\begin{array}{c}
N_{0} \\
N_{0}+1
\end{array}\right] \\
& \times \Theta\left[x\left(x \pm 1-x_{1}+x_{0}\right)\right] \\
& \times \int_{0}^{\pi / 2} d \theta \frac{\cos ^{6} \theta \sin ^{2} \theta}{D_{ \pm}\left(c_{1}, c_{2}, B_{10}, 1,0, x, \theta\right)}
\end{aligned}
$$

and

$$
\begin{aligned}
\lambda_{01}^{ \pm}(x)= & 2 \alpha b_{0} L_{0} \omega_{\mathrm{LO}} C_{0}\left[\begin{array}{c}
N_{0} \\
N_{0}+1
\end{array}\right] \\
& \times \Theta\left[x\left(x \pm 1+x_{1}-x_{0}\right)\right] \\
& \times \int_{0}^{\pi / 2} d \theta \frac{\cos ^{6} \theta \sin ^{2} \theta}{D_{ \pm}\left(c_{1}, c_{2}, B_{01}, 0,1, x, \theta\right)},
\end{aligned}
$$

with $\quad C_{0}=1.703169, \quad c_{1}=0.951559, \quad c_{2}=0.769129$, $B_{10}=\left(x_{1}-x_{0} \mp 1\right)^{2}$, and $B_{01}=\left(x_{0}-x_{1} \mp 1\right)^{2}$.

From the above expressions for the scattering rates for a Q2DEG, one notices that if the conditions $x \geq 0$ and $x \pm 1-x_{m}+x_{n} \geq 0$ are satisfied, the electron with an inplane energy $x$ in subband $n$ (with corresponding confinement energy $x_{n}$ ) will interact with a LO phonon and give a nonzero contribution to the scattering rate. The electron is scattered into subband $m$ (confinement energy $x_{m}$ ) with translational energy $x \pm 1-x_{m}+x_{n}$ by absorbing (upper sign) or emitting (lower sign) a LO phonon. There are two factors influencing the scattering rate: (1) the electron kinetic energy in the $x y$ plane and (2) the subband the electron is in. By changing the electron energy, or (and) the electric subband, one alters the allowed scattering processes.

To limit the number of parameters, we introduce the total scattering rate for an electron in a given subband $n$ :

$$
\Gamma_{n}(x)=\sum_{m} \lambda_{n m}(x)
$$

This scattering rate is plotted in Figs. 2(a)-2(c) for different subband indices $n$ as a function of the total electron energy $E=E_{n}(\mathbf{k})=E_{n}+E_{\mathbf{k}}$ for a lattice temperature of $T=220 \mathrm{~K}$. We show the results for (a) the parabolicwell case with $\omega_{0}=\omega_{\text {LO }}$, (b) the square-well case with width $L / L_{0}=\pi$ (i.e., $L=124 \AA$ for GaAs), and (c) the triangular-well case with electric field

$$
F_{s} / F_{0}=1 /\left(6 A_{0}^{3 / 2}\right)=0.28
$$

(i.e., $F_{s}=25.9 \mathrm{kV} / \mathrm{cm}$ for GaAs). Each step in the total scattering rate corresponds to the threshold of a new scattering channel, given by (1) $E / \hbar \omega_{\mathrm{LO}}=x_{n}$, which is the condition that electric subband $n$ is occupied by electrons; and (2) $E / \hbar \omega_{\mathrm{LO}}=x_{m} \mp 1$, which is the condition that electron LO-phonon emission (absorption) occurs. The steps are the finite discontinuous jumps after which the scattering rate decreases with increasing energy until the next channel opens up.

In the present paper we are interested in the electrophonon effect where the width of the Q2DEG is varied in order to bring the energy levels in resonance with the optical-phonon energy. The scattering rate of an electron in subband $n$ and with given kinetic energy

$$
E_{\mathbf{k}}=\hbar^{2} /\left(2 m^{*}\right)\left(k_{x}^{2}+k_{y}^{2}\right)
$$



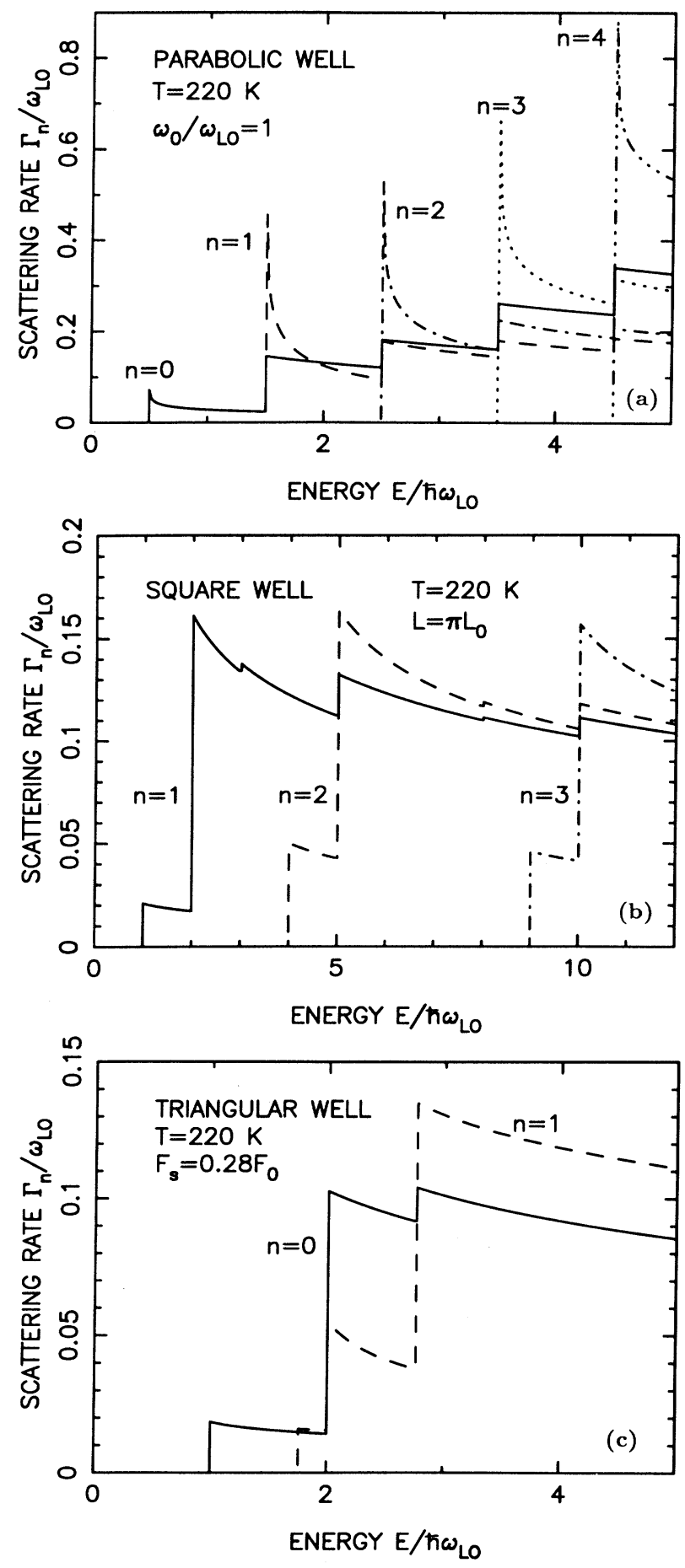

FIG. 2. The scattering rate $\Gamma_{n}=\sum_{m} \lambda_{n m}$ for an electron in subband $n$ is plotted as a function of the total electron energy $E=E_{n}+E_{k}$ at the temperature $T=220 \mathrm{~K}$ for (a) a parabolic well with a resonant frequency of harmonic oscillator $\omega_{0} / \omega_{\mathrm{LO}}=1$ for the subbands $n=0,1,2,3$, and 4 , (b) the square well with a well width $L / L_{0}=\pi$ (i.e., $L=124 \AA$ for GaAs) for the subbands $n=1,2$, and 3 , and (c) the triangular well with an electric field $F_{s} / F_{0}=1 /\left(6 A_{0}^{3 / 2}\right)=0.28$ (i.e., $F_{s}=25.9 \mathrm{kV} / \mathrm{cm}$, $A_{0}=0.708120$ for $\mathrm{GaAs}$ ) for the subbands $n=0$ and 1 . For GaAs, $\hbar \omega_{\mathrm{LO}}=36.6 \mathrm{meV}, \quad \omega_{\mathrm{LO}}=5.56 \times 10^{13} \mathrm{~s}^{-1}, \quad$ and $m^{*} / m_{e}=0.0665$. is depicted in Figs. 3(a)-3(c) for (i) $E_{\mathrm{k}}=0$ and (ii) a nonzero kinetic energy $\left(E_{\mathbf{k}}=\hbar \omega_{\mathrm{LO}}\right)$ as a function of the width of the Q2DEG for $T=220 \mathrm{~K}$ and the three different confinement potentials. For a triangular well, increasing the electric field implies decreasing the width of the 2DEG. This is similar to the parabolic potential case when increasing the confinement frequency $\omega_{0}$ implies decreasing the width $L \sim 1 / \sqrt{\omega_{0}}$. In order to understand these figures, we remark that the electron state $|\mathbf{k}, n\rangle$ can be changed in three possible ways after interacting with a LO phonon: (1) the electron can stay in subband $n$ and gain (lose) an energy $\hbar \omega_{\text {LO }}$ by absorption (emission) (for $x=E / \hbar \omega_{\mathrm{LO}}<1$, the emission process is not possible), (2) the electron can be scattered to a lower subband $m$ and gain an energy $x_{n}-x_{m} \pm 1$ (for $x<x_{m}+1-x_{n}$ the LO-phonon emission process is not possible), and (3) scatter to a higher subband $m$ and lose an energy $x_{m}-x_{n} \mp 1$. This process is only possible when $x>x_{m}-x_{n} \mp 1$. For cases (2) and (3) the energy difference between the two subbands is of importance.

When the electron kinetic energy is smaller than a LO phonon, i.e., $x<1$, the electron LO-phonon interaction, as shown in Figs. 3(a)-3(c), strongly depends on the energy difference between the two subbands. The step increase (decrease) in Figs. 3(a)-3(c) corresponds to the opening up (closing) of a scattering channel by changing these parameters. Notice that the total scattering rate increases (decreases), especially for lower subbands, with increasing energy difference between the two subbands when $\left|x_{m}-x_{n}\right|$ is smaller (larger) than 1. At the point $\left|x_{m}-x_{n}\right|=1$, the scattering rate $\Gamma_{n}$ exhibits a step decrease. The reason is that when $\left|x_{m}-x_{n}\right|<1$, the enhancement of the energy difference between two subbands is equivalent to a suppression of the electron kinetic energy, which leads to an increase of the total scattering rate, while for the case of $\left|x_{m}-x_{n}\right|>1$, an enhancing energy difference between the two subbands is equivalent to an increase of the electron kinetic energy.

When the electron kinetic energy $x \geq 1$, the steps in $\Gamma_{n}$ are relatively weak. This is illustrated in Figs. 3(a)-3(c) for $E_{\mathbf{k}}=\hbar \omega_{\text {LO }}$. Note from Figs. 3(a)-3(c) that in this case the total scattering rate always keeps increasing with increasing value of the electric subband index $n$, except at the step change which corresponds to $\left|E_{m}-E_{n}\right|=2 \hbar \omega_{\text {LO }}$ where a scattering channel opens up for electrons in subband $n$ which is able to absorb a LO phonon and go to a higher subband $m$.

\section{THE ELECTROPHONON RESONANCE EFFECT IN THE CONDUCTIVITY}

In the relaxation-time approximation, the conductivity is given by ${ }^{25,26}$

$$
\sigma=-\frac{e^{2}}{\Omega} \sum_{n, \mathbf{k}} \frac{\partial f_{n}\left(E_{\mathbf{k}}\right)}{\partial E_{\mathbf{k}}} \frac{v_{x}^{2}}{\Gamma_{n}(\mathbf{k})},
$$

where $f_{n}\left(E_{\mathbf{k}}\right)$ is the electron energy distribution function for the electric subband $n, \Gamma_{n}(\mathbf{k})$ the total scattering rate for an electron in subband $n$ with momentum $\mathbf{k}$, and $v_{x}=\hbar k_{x} / m^{*}$ the electron average velocity along the elec- 

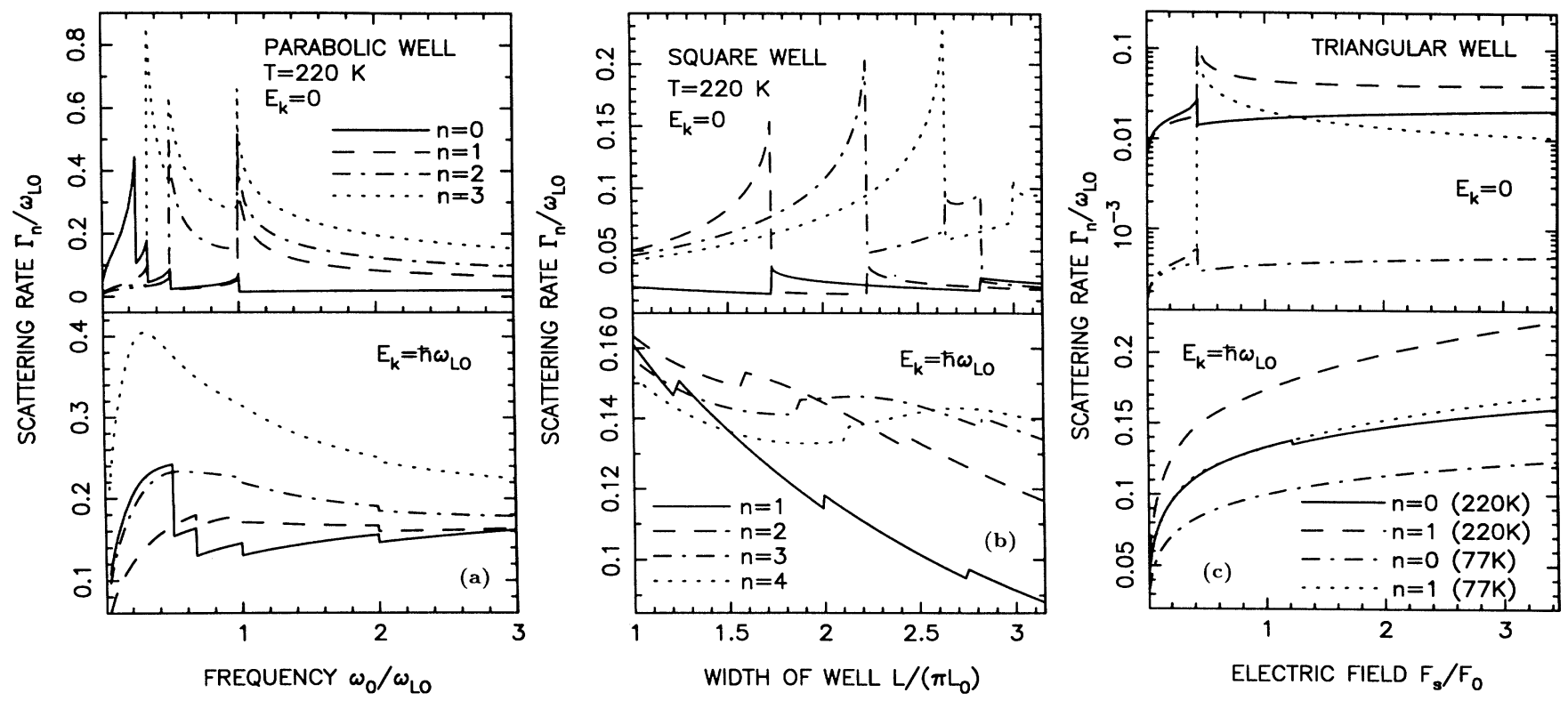

FIG. 3. For $E_{k}=\hbar^{2} /\left(2 m^{*}\right)\left(k_{x}^{2}+k_{y}^{2}\right)=0$ and $=\hbar \omega_{\mathrm{LO}}$ in-plane kinetic energy; the total scattering rate for electrons in a subband $n$ are plotted as function of (a) the resonant frequency of the harmonic oscillator at a temperature $T=220 \mathrm{~K}$ for the subbands $n=0,1$, 2, 3, and 4 for the parabolic-well case; (b) the width of the quantum well at a temperature $T=220 \mathrm{~K}$ for the subbands $n=1,2,3$, and 4 for the square-well case; and (c) the electric field at the temperatures $T=77$ and $220 \mathrm{~K}$ and for the subbands $n=0$ and 1 for the triangular-well case. For GaAs, $L_{0}=39.5 \AA, F_{0}=92.5 \mathrm{kV} / \mathrm{cm}$.

tric field. For the nondegenerate case and at relatively high temperature, $f_{n}\left(E_{\mathbf{k}}\right)$ is a Maxwellian distribution,

$$
f_{n}\left(E_{\mathbf{k}}\right)=\frac{\hbar^{2} \beta}{2 \pi m^{*}} e^{-\beta E_{n}} e^{-\beta E_{\mathbf{k}}} / \sum_{n} e^{-\beta E_{n}}
$$

with $\beta=1 / k_{B} T$. In this case the conductivity becomes

$$
\frac{\sigma}{\sigma_{0}}=\frac{\beta_{0}^{2}}{Z_{0}} \sum_{n} e^{-\beta_{0} E_{n} / \hbar \omega_{\mathrm{LO}}} \int_{0}^{\infty} d x x e^{-\beta_{0} x} \frac{\omega_{\mathrm{LO}}}{\Gamma_{n}(x)},
$$

where $Z_{0}=\Sigma_{n} e^{-\beta_{0} E_{n} / \hbar \omega_{\mathrm{LO}}} ; \sigma_{0}=e^{2} /\left(4 \pi^{2} m^{*} \omega_{\mathrm{LO}} \Omega\right)$, with $\Omega$ the surface area; $\beta_{0}=\hbar \omega_{\mathrm{LO}} / k_{B} T$; and $x=E_{k} / \hbar \omega_{\mathrm{LO}}$.

The integral in Eq. (12) has to be calculated numerical1y. Special care has to be taken at the discontinuities in the total scattering rate $\Gamma_{n}(x)=\sum_{n} \lambda_{n m}(x)$. Therefore, the conductivity is written as

$$
\frac{\sigma}{\sigma_{0}}=\frac{\beta_{0}^{2}}{Z_{0}} \sum_{n} e^{-\beta_{0} E_{n} / \hbar \omega_{\mathrm{LO}}} \sum_{i} \int_{a_{i}}^{a_{i+1}} d x x e^{-\beta_{0} x} \frac{\omega_{\mathrm{LO}}}{\Gamma_{n}(x)}
$$

where $0 \leq a_{i}<a_{i+1}$ and

$$
a_{i}= \begin{cases}0, & \text { initial } \\ 1, & n=m \\ \left(E_{m}-E_{n}\right) / \hbar \omega_{\mathrm{LO}} \mp 1, & n \neq m .\end{cases}
$$

Equation (13) involves the numerical calculation of an integral which makes this expression less transparent. For this purpose it is instructive to approximate the total scattering rate $\Gamma_{n}(x)$ by a constant $\Gamma_{n}\left(a_{i}\right)=\Sigma_{m} \lambda\left(a_{i}\right)$ for each scattering process. In so doing, the energy dependence of a specific scattering process is neglected. In this case the conductivity then takes the simple form

$$
\begin{aligned}
& \frac{\sigma}{\sigma_{0}}=\frac{\beta_{0}}{Z_{0}} \sum_{n} e^{-\beta_{0} E_{n} / \hbar \omega_{\mathrm{LO}}} \\
& \times \sum_{i} \frac{\omega_{\mathrm{LO}}}{\Gamma_{n}\left(a_{i}\right)}\left[e^{-\beta_{0} a_{i}}\left(a_{i}+\frac{1}{\beta_{0}}\right)\right. \\
&\left.-e^{-\beta_{0} a_{i+1}}\left(a_{i+1}+\frac{1}{\beta_{0}}\right)\right] .
\end{aligned}
$$

A numerical evaluation of Eqs. (13) and (14) shows that in the simple expression (14) the electrophonon-resonance effect is more pronounced than in the nonapproximated expression (13). Therefore, in the following numerical results we show the results corresponding to Eq. (13).

The numerical results for the conductivity and its first derivative are shown in Fig. 4 for (a) the parabolic well as a function of $\omega_{0} / \omega_{\mathrm{LO}}$, (b) the square well as a function of the width $L / L_{0}$, and (c) the triangular well as a function of the electric field $F_{s} / F_{0}$ for four different temperatures $T=77,100,140$, and $220 \mathrm{~K}$. Note the following.

(1) The conductivity oscillates as a function of the separation between the electric subbands. For the triangular-well case, only the two lowest subbands are taken into account, and therefore only one oscillation is found.

(2) When the energy difference between two subbands 

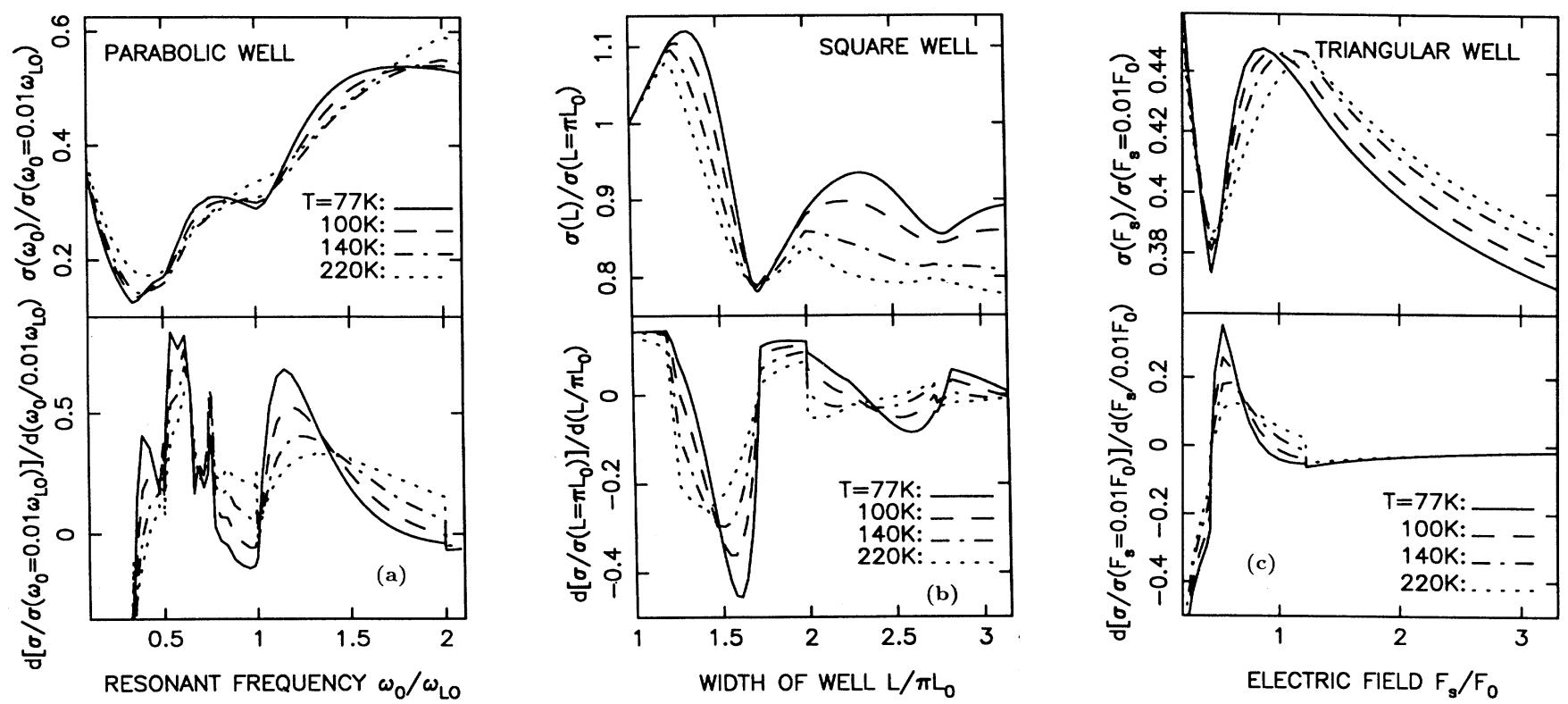

FIG. 4. The conductivity and the first derivative of the conductivity are plotted as a function of (a) $\omega_{0} / \omega_{\mathrm{LO}}$, the resonant frequency for the parabolic-well case; (b) $L / L_{0}$, the width of the well for the square-well case; and (c) $F_{s} / F_{0}$, the electric field for the triangular-well case, at the temperatures $T=77,100,140$, and $220 \mathrm{~K}$. In (a) the subbands $n=0,1,2,3$, and 4 are included, and the conductivities are scaled by their values at $\omega_{0}=0.01 \omega_{\mathrm{LO}}$. In (b) the subbands $n=1,2,3,4$, and 5 are included, and the conductivities are scaled by their values at $L=\pi L_{0}$. In (c) the subbands $n=0$ and 1 are included, and the conductivity are scaled by their values at $F_{s}=0.01 F_{0}$.

is smaller (larger) than the LO-phonon energy $\hbar \omega_{\mathrm{LO}}$, the conductivity decreases (increases) with increasing the energy difference between the subbands. The conductivity attains a local minimum when the energy difference between two subbands equals $\hbar \omega_{\text {LO }}$.

(3) The electrophonon-resonance effect is most pronounced when the two lowest subbands are in resonance. This is most clearly shown for the square-well case [Fig. 4(b)]. This is due to the $n^{2}$ dependence of the subband energy. For the parabolic-well case, shown in Fig. 4(a), the electrophonon-resonance effect between the higher subbands, i.e., $N \omega_{0}=\omega_{\text {LO }}$, also shows up in the conductivity because of the linear dependence of the subband energy on the level number $n$.

(4) The first derivative of the conductivity shows the electrophonon resonance in a more pronounced way. The step changes in the derivative of the conductivity correspond to the step changes in the total scattering rate. Note that in Figs. 4(a)-4(c) the resonance amplitude decreases with increasing temperature, which is opposite to the temperature dependence found for magnetophonon resonances. The physical reason behind this will be given below.

The influence of the absorption and emission scattering processes on the electrophonon-resonance effect in the conductivity can be studied by considering the situation with lattice temperatures $T=4.2 \mathrm{~K}$ and electron temperatures $T_{e}=77,100,140$, and $220 \mathrm{~K}$, such that the LOphonon occupation number $N_{0} \simeq 0$ and only the LOphonon emission process is possible. After inserting $\beta_{0}=\hbar \omega_{\mathrm{LO}} / k_{B} T_{e}$ into Eq. (13), we found that the numeri- cal results for the scaled conductivity $\left[\sigma\left(\omega_{0}\right) / \sigma\left(\omega_{0}=0.01 \omega_{\mathrm{LO}}\right), \quad \sigma(L) / \sigma\left(L=\pi L_{0}\right), \quad\right.$ and $\left.\sigma\left(F_{s}\right) / \sigma\left(F_{s}=0.01 F_{0}\right)\right]$ practically coincide with the results in the case of $T=T_{e}$. Thus the emission scattering process is of paramount importance in the electrophonon-resonance effect on the conductivity. And

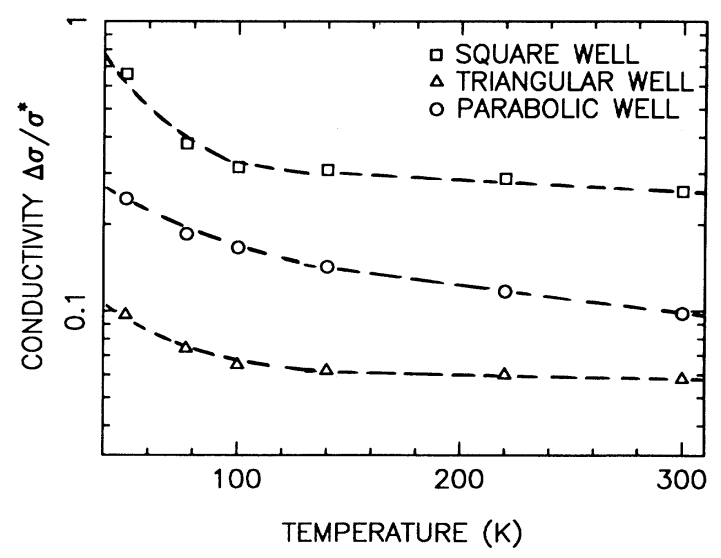

FIG. 5. The amplitude of the conductivity as a function of temperature for three kinds of confinement potentials. $\Delta \sigma=\sigma_{\max }-\sigma_{\min }$, where $\sigma_{\max }\left(\sigma_{\min }\right)$ is the first maximum (minimum) value of the conductivity in Fig. 4. $\sigma^{*}=\sigma\left(L=\pi L_{0}\right)$ (for a square well), $\sigma^{*}=\sigma\left(F_{s}=0.01 F_{0}\right)$ (for a triangular well), and $\sigma^{*}=\sigma\left(\omega_{0}=0.01 \omega_{\mathrm{LO}}\right)$ (for a parabolic well). The dashed curves are guides to the eye. 
the absorption scattering (in the case of $T=T_{e}$ ) only leads to an overall reduction of the conductivity. The emission scattering rate is much more strongly influenced by changing the strength of the confinement potential, as is apparent from Figs. 2 and 3. Thus the emission scattering process is the main contributor to the electrophonon-resonance effect in the conductivity and this is also the reason why the electrophonon resonances are more pronounced at low temperature. With increasing temperature, the absorption scattering rate increases, which leads to an enhanced background scattering rate and to "relative" smaller electrophonon resonance. Also the thermal broadening of the electron energy distribution contributes to the smoothing of the resonances. Notice that for the electrophonon resonance-effect the density of state (DOS) has only a steplike behavior which has to be compared with the singular nature of the DOS in the case of the magnetophonon resonances. In the latter the thermal broadening is more important and consequently leads to a decreasing magnetophonon-resonance amplitude with increasing temperature. The amplitude of the electrophonon resonance in the conductivity $\left[\Delta \sigma=\sigma_{\max }-\sigma_{\min } \quad\right.$ and $\sigma^{*}=\sigma\left(L=\pi L_{0}\right), \quad \sigma^{*}=\sigma\left(F_{s}\right.$ $\left.=0.01 F_{0}\right), \sigma^{*}=\sigma\left(\omega_{0}=0.01 \omega_{\text {LO }}\right)$ in Fig. 4] is plotted in Fig. 5 as a function of temperature for the three different confinement potentials.

\section{SUMMARY AND CONCLUSIONS}

In conclusion, we have studied the electrophonon resonance-effect in a quasi-two-dimensional electron system in the absence of any magnetic field. By increasing the energy difference between two electric subbands $n$ and $m$, the total scattering rate for an electron with an energy component $E_{k}<\hbar \omega_{\text {LO }}$ in subband $n$ increases (decreases) when the energy difference between two subbands $\left|E_{m}-E_{n}\right|$ is smaller (larger) than the LO-phonon energy $\hbar \omega_{\text {LO }}$. When $\left|E_{m}-E_{n}\right|=\hbar \omega_{\text {LO }}$, resonant scattering occurs which leads to a step change in the scattering rate and this will influence the electric properties of the 2DEG. The conductivity was calculated in order to ob- serve this effect. When the energy difference between subbands $m$ and $n,\left|E_{m}-E_{n}\right|$, is smaller (larger) than $\hbar \omega_{\text {LO }}$, the conductivity decreases (increases) when this energy difference increases. At the point $\left|E_{m}-E_{n}\right|=\hbar \omega_{\text {LO }}$, the conductivity has a local minimum. In the present paper we studied the electrophonon-resonance effect by considering three kinds of confinement potentials: (1) the parabolic well, (2) the square well, and (3) the triangular well.

We found that the electron LO-phonon emission scattering is of paramount importance for the electrophonon-resonance effect in the conductivity. The amplitude of the oscillation decreases with increasing temperature, which is opposite to the related magnetophonon-resonance effect. The most dominant resonance occurs for the lowest electrical quantum level. Higher subbands lead only to small contributions to the resonance structure.

The electrophonon-resonance effect was measured in Ref. 5 using a graded quantum well. Unfortunately, the measurements were not sufficiently systematic in order to allow for a detailed comparison with our theoretical calculation. We propose a similar experiment in which the gate voltage is modulated such that the derivative of the conductivity is immediately obtained. In this way the electrophonon-resonance effect would show up in a much more pronounced way.

In the present calculation for the conductivity, we took the most simple situation of linear transport and a nondegenerate electron gas. The nonlinear electrophononresonance effects will be studied in a separate paper.

\section{ACKNOWLEDGMENTS}

F.M.P. is supported by the Belgian National Science Foundation and W.X. by the Supercomputer Project of the NFWO (Nationaal Fonds voor Wetenschappelijk Onderzoek), Belgium. F.M.P. acknowledges discussions with A. Kastalsky and J. P. Leburton.
*Also at University of Antwerp (RUCA), B-2020 Antwerpen and Technical University Eindhoven, NL-5600 Eindhoven, The Netherlands.

${ }^{1}$ See, e.g., Two-Dimensional System, Heterostructures, and Superlattices, edited by G. Bauer, F. Kuchar, and H. Heinrich, Solid-State Sciences Vol. 53 (Springer-Verlag, Berlin, 1984).

${ }^{2}$ X. G. Wu, F. M. Peeters, and J. T. Devreese, Phys. Rev. B 31, 3420 (1985); Phys. Status Solidi B 133, 229 (1986).

${ }^{3}$ F. M. Peeters and J. T. Devreese, Phys. Rev. B 36, 4442 (1987).

${ }^{4}$ S. Das Sarma, Phys. Rev. B 27, 2590 (1983); 31, 4034(E) (1985); F. M. Peeters, X. G. Wu, and J. T. Devreese, Solid State Commun. 65, 1505 (1988); F. M. Peeters, X. G. Wu, J. T. Devreese, C. J. G. M. Langerak, J. Singleton, D. J. Barnes, and R. J. Nicholas, Phys. Rev. B 45, 4296 (1992).

${ }^{5}$ A. Kastalsky, F. M. Peeters, W. K. Chan, L. T. Florez, and J. P. Harbison, Appl. Phys. Lett. 59, 1708 (1991).

${ }^{6}$ F. M. Peeters and J. T. Devreese, in Proceedings of the Seventh
International Conference on Hot Carriers in Semiconductors, July 1991, Nara, Japan [Semicond. Sci. Technol. 7, B15 (1992)].

${ }^{7}$ V. L. Gurevich and Yu. A. Firsov, Zh. Eksp. Teor. Fiz. 13, 199 (1961) [Sov. Phys. - JETP 13, 137 (1961).

${ }^{8}$ For a recent review see, e.g., R. T. Nicholas, Prog. Quantum Electron 10, 1 (1985).

${ }^{9}$ S. Komiyama, H. Eyferth, and J. P. Kotthaus, J. Phys. Soc. Jpn. 49, Suppl. A, 687 (1980).

${ }^{10}$ P. T. Coleridge, Semicond. Sci. Technol. 5, 961 (1990).

${ }^{11}$ M. I. Kaganov, S. S. Nedorerov, and A. M. Rustamova, Fiz. Tverd. Tela (Leningrad) 12, 2277 (1970) [Sov. Phys.-Solid State 12, 1820 (1977)].

${ }^{12}$ P. Warmembol, F. M. Peeters, and J. T. Devreese, Phys. Rev. B 37, 4694 (1988).

${ }^{13}$ W. Cai, X. L. Lei, and C. S. Ting, Phys. Rev. B 31, 4070 (1985). 
${ }^{14}$ H. L. Stormer, A. C. Gossard, W. Wiegmann, and K. Baldwin, Appl. Phys. Lett. 39, 912 (1981).

${ }^{15}$ S. Briggs and J. P. Leburton, Superlatt. Microstruc. 5, 145 (1989); S. Briggs, D. Jovanovic, and J. P. Leburton, Appl. Phys. Lett. 54, 2012 (1989); Solid-State Electron. 32, 1657 (1989).

${ }^{16}$ F. M. Peeters, A. Kastalsky; W. Xu, and J. T. Devreese (unpublished).

${ }^{17}$ B. K. Ridley, J. Phys. C 15, 5899 (1982); F. A. Riddoch and B. K. Ridley, ibid. 16, 6971 (1983).

${ }^{18}$ See, e.g., A. Yariv, An Introduction to Theory and Applications of Quantum Mechanics (Wiley, New York, 1982).

${ }^{19}$ P. J. Price, Ann. Phys. (N.Y.) 133, 261 (1981).

${ }^{20}$ T. Ando, A. B. Fowler, and F. Stern, Rev. Mod. Phys. 54, 437
(1982).

${ }^{21}$ Handbook of Mathematical Functions, edited by $\mathbf{M}$. Abramowitz and I. A. Stegun, Natl. Bur. Stand. Appl. Math. Ser. No. 55 (GPO, Washington, 1964).

${ }^{22}$ T. S. Rahman, D. L. Mills, and P. S. Riseborough, Phys. Rev. B 23, 4081 (1981).

${ }^{23}$ P. J. Price, Surf. Sci. 113, 199 (1982).

${ }^{24}$ W. Walukiewicz, H. E. Ruda, J. Iagowski, and H. C. Gatos, Phys. Rev. B 30, 4571 (1984); F. F. Fang and W. E. Howard, Phys. Rev. Lett. 16, 797 (1966).

${ }^{25}$ F. M. Peeters and J. T. Devreese, Solid State Phys. 38, 81 (1984).

${ }^{26}$ V. K. Arora and F. G. Arad, Phys. Rev. B 23, 5570 (1981). 